\title{
Recognition of the Lipoyl Domain is the Ultimate Determinant of Substrate Channelling in the Pyruvate Dehydrogenase Multienzyme Complex
}

\author{
D. Dafydd Jones, Katherine M. Stott, Pedro A. Reche \\ and Richard N. Perham*
}

Cambridge Centre for

Molecular Recognition

Department of Biochemistry,

University of Cambridge, 80

Tennis Court Road, Cambridge

CB2 1GA, UK

\begin{abstract}
Reductive acetylation of the lipoyl domain (E2plip) of the dihydrolipoyl acetyltransferase component of the pyruvate dehydrogenase multienzyme complex of Escherichia coli is catalysed specifically by its partner pyruvate decarboxylase (E1p), and no productive interaction occurs with the analogous 2-oxoglutarate decarboxylase (E1o) of the 2-oxoglutarate dehydrogenase complex. Residues in the lipoyl-lysine $\beta$-turn region of the unlipoylated E2plip domain (E2plip ${ }^{\text {apo }}$ ) undergo significant changes in both chemical shift and transverse relaxation time $\left(T_{2}\right)$ in the presence of E1p but not E1o. Residue Gly11, in a prominent surface loop between $\beta$-strands 1 and 2 in the E2plip domain, was also observed to undergo a significant change in chemical shift. Addition of pyruvate to the mixture of E2plip ${ }^{\text {apo }}$ and E1p caused larger changes in chemical shift and the appearance of multiple cross-peaks for certain residues, suggesting that the domain was experiencing more than one type of interaction. Residues in both $\beta$-strands 4 and 5 , together with those in the prominent surface loop and the following $\beta$-strand 2, appeared to be interacting with E1p, as did a small patch of residues centred around Glu31. The values of $T_{2}$ across the polypeptide chain backbone were also lower than in the presence of E1p alone, suggesting that E2plip ${ }^{\text {apo }}$ binds more tightly after the addition of pyruvate. The lipoylated domain (E2plipholo) also exhibited significant changes in chemical shift and decreases in the overall $T_{2}$ relaxation times in the presence of E1p, the residues principally affected being restricted to the half of the domain that contains the lipoyl-lysine (Lys41) residue. In addition, small chemical shift changes and a general drop in $T_{2}$ times in the presence of E1o were observed, indicating that E2plip ${ }^{\text {holo }}$ can interact, weakly but non-productively, with E1o. It is evident that recognition of the protein domain is the ultimate determinant of whether reductive acetylation of the lipoyl group occurs, and that this is ensured by a mosaic of interactions with the Elp.
\end{abstract}

Keywords: pyruvate dehydrogenase complex; lipoyl domain; lipoylation; protein-protein interaction

Present addresses: D. D. Jones, MRC Centre for Protein Engineering, Hills Road, Cambridge, CB2 2QH, UK; P. A. Reche, DNAX Research Institute, 901 California Ave, Palo Alto, CA 94304-1104, USA.

Abbreviations used: E1o, 2-oxoglutarate decarboxylase; E1p, pyruvate decarboxylase; E2p, dihydrolipoyl acetyltransferase; E2plip, innermost lipoyl domain of E. coli E2p; E2plip ${ }^{\text {apo }}$, unlipoylated domain; E2plip ${ }^{\text {holo }}$, lipoylated domain; HSQC, heteronuclear single quantum correlation; LplA, lipoyl protein ligase A; NMR, nuclear magnetic resonance; $\mathrm{PDH}$, pyruvate dehydrogenase; $T_{2}$, transverse relaxation time; OGDH, 2-oxoglutarate; $\mathrm{BCDH}$, branched-chain 2-oxo acid; BSA, bovine serum albumin.

E-mail address of the corresponding author: r.n.perham@bioc.cam.ac.uk 


\section{Introduction}

The pyruvate dehydrogenase (PDH) complex catalyses the oxidative decarboxylation of pyruvate, transferring the resultant acetyl group to coenzyme A. It belongs to a family of related 2-oxo acid dehydrogenase complexes, which includes the 2-oxoglutarate (OGDH) and branched-chain 2-oxo acid $(\mathrm{BCDH})$ dehydrogenase complexes. In the PDH complex, the three component enzymes are pyruvate decarboxylase (E1p; EC 1.2.4.1), dihydrolipoyl acetyltransferase (E2p; EC 2.3.1.12) and dihydrolipoyl dehydrogenase (E3; EC 1.8.1.4) (reviewed by Perham, 1991, 2000; de Kok et al., 1998). The pyruvate decarboxylase catalyses the initial decarboxylation of the 2-oxo acid, using thiamin diphosphate (ThDP) as a cofactor, and the subsequent reductive acetylation of a lipoyl group bound to the E2p. E2p catalyses the transfer of the acetyl group to CoA, and E3 concludes the process by reoxidizing the dihydrolipoyl group to regenerate the dithiolane ring, with $\mathrm{NAD}^{+}$as the final electron acceptor. In the PDH complex from Escherichia coli and most Gram-negative bacteria, E2p forms a cubic core consisting of 24 polypeptide chains arranged with octahedral symmetry, whereas in Bacillus stearothermophilus and most Gram-positive bacteria, the E2p core is icosahedral and comprises 60 E2p chains (Reed \& Hackert 1990; Perham, 1991). E1p and E3 are bound tightly but non-covalently around the core. The OGDH and $\mathrm{BCDH}$ complexes follow the same structural pattern.

The lipoyl group is covalently attached in an amide linkage to the $N^{6}$-amino group of a specific lysine residue of an independently folded domain that forms the N-terminal part of the E2p chain. In the E2p chain of E. coli there are three such lipoyl domains, whereas in that of B. stearothermophilus and the E2o chain of E. coli there is only one (Reed \& Hackert, 1990; Perham 1991). This lipoyl domain plays a vital role in coupling the reactions within the complex in an organized and specific manner (Perham, 1991, 2000; de Kok et al., 1998). Acting as a "swinging arm" (Reed, 1974), the lipoyl group visits each of the three active sites of the complex, carried by a mobile lipoyl domain that protrudes out from the inner core of the complex.

The nuclear magnetic resonance (NMR) solution structures of the single E. coli E2o (Ricaud et al., 1996) and the innermost of the three E. coli E2p (Jones et al., 2000a) lipoyl domains have been solved, as have those of others from various other organisms (Dardel et al., 1993; Berg et al., 1996, 1997; Howard et al., 1998). Their overall backbone structure is virtually identical, comprising a $\beta$-barrel formed from two four-stranded $\beta$-sheets. The $\beta$-sheets have a 2 -fold axis of quasi-symmetry, with the lipoyl-lysine residing at the tip of a tight, type I $\beta$-turn. Positioning of the target lysine at its exact location within the $\beta$-turn is essential for correct post-translational modification (Wallis \& Perham, 1994). A prominent surface loop, linking $\beta$-strands 1 and 2 , is only present in one of the $\beta$-sheets and lies close in space to the lipoyllysine $\beta$-turn. This loop is important for the structure, post-translational modification and catalytic function of the domain (Wallis et al., 1996; Jones et al., $2000 \mathrm{a}, \mathrm{b})$.

Attachment of the lipoic acid to the lipoyl domain occurs in two ways (Morris et al., 1994; Green et al., 1995; Jordan \& Cronan, 1997a). In the reaction catalysed by lipoyl protein ligase A (LplA), activation of the carboxyl group of lipoic acid by reaction with ATP to form lipoyl-AMP is followed by transfer of the lipoyl group to the target lysine residue and the release of AMP. An alternative ATP-independent reaction is thought to involve lipoic acid attached to the acyl carrier protein of fatty acid synthesis as the activated intermediate (Jordan \& Cronan, 1997a,b). Lipoyl domains from a wide range of organisms and 2-oxo acid dehydrogenase complexes, together with the H-protein from the glycine cleavage system, can be lipoylated by the E. coli lipoylating machinery (Dardel et al., 1991; Quinn et al., 1993; Berg et al., 1994, 1995; Wallis \& Perham, 1994; Macherel et al., 1996), but the lipoyl domain of the bovine BCDH complex is an exception (Griffin et al., 1990).

With the E. coli PDH complex, free lipoate can act as the substrate for E2p and E3 but is a very poor substrate for E1p. However, the E2p lipoyl domain is an excellent substrate $\left(k_{\text {cat }} / K_{\mathrm{m}}\right.$ raised by a factor of $\left.10^{4}\right)$. Moreover, the E2p and E2o lipoyl domains from E. coli (Graham et al., 1989; Jones et al., 2000b) function as substrates only for their cognate E1 s. Similar results have been reported for the E2p and E2o lipoyl domains of Azotobacter vinelandii (Berg et al., 1998). Thus, the lipoyl domain provides an elegant mechanism for substrate channelling such that reductive acylation is confined to a lipoyl group covalently attached to a specific lysine residue of the intended E2 component (Perham, 1991, 2000; de Kok et al., 1998). The question arises as to how the E. coli E2p and E2o lipoyl domains display such distinct activities towards their cognate E1, yet possess an identical structural scaffold. The binding of the lipoyl domain to E. coli E1p is weak $\left(K_{\mathrm{s}}\right.$ not less than $1 \mathrm{mM}$, despite a $K_{\mathrm{m}}$ of ca $20 \mu \mathrm{M}$ ) and transient (Graham \& Perham, 1990). Thus co-crystallization of the lipoyl domain with E1 is likely to be impossible. However, NMR has been used to identify regions on the apo-form of the B. stearothermophilus E2p lipoyl domain that undergo an interaction with E1p. Alterations in both chemical shift (Wallis et al., 1996) and transverse $\left(T_{2}\right)$ relaxation times (Howard et al., 2000) have been utilized for this purpose. It is surprisingly that the backbone amide of the lysine to which the lipoic acid is attached does not appear to undergo an interaction with E1p.

The quaternary structures of the E. coli $\left(\alpha_{2}\right)$ and B. stearothermophilus $\left(\alpha_{2} \beta_{2}\right)$ E1p differ, and therefore so may their interactions with the lipoyl domain. The crystal structure of an $\alpha_{2} \beta_{2}$ E1p from Pseudomo- 
nas putida has recently been solved (Ævarsson et al., 1999). The ThDP in the active site is buried at the bottom of a $20 \AA$ deep funnel-shaped hole at the interface between the $\alpha$ and $\beta$ subunits; in order for the lipoyl group to reach the cofactor site, assuming that no major conformational changes ensue, the lipoyl domain has to come into close contact with the Elp surface and the lipoyl-lysine side-chain must become fully extended. Here, we use NMR to investigate regions of the innermost lipoyl domain of E. coli E2p that interact with the E. coli $\alpha_{2}$ E1p. We analyse the interaction of the holo-domain (E2plip ${ }^{\text {holo }}$ ) and the apo-domain $\left(\mathrm{E}^{2} \mathrm{plip}^{\mathrm{apo}}\right)$ with Elp, and the catalytically unproductive interaction of the E2plip apo and E2plipholo domains with $E$. coli Elo. It has been reported that the $\alpha_{2}$ E1p from A. vinelandii is more resistant to proteolysis in the presence of pyruvate (Hengeveld et al., 1997) and that pyruvate decreases ThDP dissociation from E. coli Elp by a factor of 10 (Hennig et al., 1997), suggesting a conformational change in Elp in the presence of substrate. We have therefore also measured chemical shift and $T_{2}$ changes after the addition of pyruvate to the mixture of E. coli E2plip ${ }^{\text {apo }}$ and E1p.

\section{Results}

\section{Interaction of E2plip ${ }^{\text {apo }}$ with E1p}

Accompanying a protein-protein interaction, chemical shifts of nuclei are altered as a result of a change in the local electronic environment that differentially shields nuclei from the static magnetic field. Any conformational change that arises from complex formation can further perturb the chemical shift. When the protein-ligand interaction is weak (fast exchange), as with E1p and the lipoyl domain, a weighted "average" chemical shift is seen rather than two distinct shifts corresponding to the bound and unbound forms. In addition, the resonances derived from the bound form of the domain would be too broad to detect, given the high molecular mass of E1 (200 kDa per dimer).

Howard et al. (2000) have shown that it is also possible to use backbone ${ }^{15} \mathrm{~N}_{2}$ experiments to analyse interactions of the B. stearothermophilus lipoyl domain with its E1p. Matsuo et al. (1999) have used the same method to analyse the interaction between components of the Methylococcus capsulatus methane mono-oxygenase system. The rationale behind their approach is based on the observation that the two interacting components are in fast exchange with respect to chemical shift, but slow exchange with respect to $T_{2}$. This is clearly the case in the system described here, where titration with the Elp component leads to a progressive change in chemical shifts but all backbone resonances remain observable. Under these conditions the observed $T_{2}$ is modified according to the equation (Dwek, 1973; Jardetsky \& Roberts, 1981; Lian \& Roberts, 1993):

$$
{ }^{1} / T_{2 \text { interaction }}={ }^{1} / T_{2 \text { free }}+{ }^{1} / t_{\text {lip }}
$$

where $T_{2}$ free is the $T_{2}$ for the lipoyl domain alone, $T_{2}$ interaction is the measured $T_{2}$ in the presence of the second component, and $t_{\text {lip }}$ is the lifetime of the lipoyl domain in the free state. From this, it would be expected that all $T_{2}$ values would be reduced to some extent, as governed by $t_{\text {lip }}$. However, certain residues may undergo a reduction in $T_{2}$ greater than that experienced by the bulk backbone. This can be attributed to an additional exchange contribution, since the residues which directly contact E1 experience larger chemical shift changes on binding than the rest of the backbone. The applicability of equation (1) to this and similar systems is discussed in full by Howard et al. (2000). In order to relate the $T_{2}$ values measured for the free E2plip domain to those measured in the presence of E1, the $T_{2}$ ratio was calculated for each residue:

$$
T_{2} \text { ratio }=T_{2 \text { E2plip }} / T_{2 \text { E2plip }+ \text { E1 }}
$$

The changes in chemical shift observed in the presence of E1p were very small (Figure 1). The largest changes were centred around the lipoyllysine $\beta$-turn region. With respect to ${ }^{1} \mathrm{H}$ nuclei, Ala42, Ser43 and, to a lesser extent, Gly11, underwent a significant $\Delta \delta$. With respect to ${ }^{15} \mathrm{~N}$ nuclei, Lys41 (the target for lipoylation) and, to a lesser extent, Asp40, Ser43, Met44, Glu45 and Val46 underwent a significant $\Delta \delta$. The largest $\Delta \delta$ for the ${ }^{1} \mathrm{H}$ and ${ }^{15} \mathrm{~N}$ nuclei were $0.013 \mathrm{ppm}$ (Ala42) and 0.068 ppm (Lys41), respectively.

In the presence of either E1o or bovine serum albumin (BSA), the resonances associated with E2plip apo did not appear to undergo any significant change in chemical shift (data not shown). This strongly suggests that the chemical shift changes observed in the presence of E1p are significant and not due simply to adventitious changes (in $\mathrm{pH}$, non-specific interactions, etc.) on addition of the second component.

The E2plipapo $T_{2}$ values also indicate that the lipoyl-lysine $\beta$-turn region interacts with $\mathrm{E} 1 \mathrm{p}$ (Figure 1(c)). In general, the $T_{2}$ values decreased in the presence of E1p, consistent with a global line broadening that accompanies formation of a transient complex. Over and above this general trend, five residues underwent a significant change due to secondary effects: Lys41, Ala42, Ser43 and, to a lesser extent, Asp40 and Met44. In the presence of either E1o or BSA, $T_{2}$ values for E2plip apo did not show any significant change (Figure $1(\mathrm{~d})$ and (e)). There was a slight decrease in the overall $T_{2}$ values in the presence of E1o, but this could be attributed to an increase in viscosity.

\section{The effect of 2-oxo acid on the interaction of E2plip ${ }^{\text {apo }}$ with E1p}

Addition of the substrate, pyruvate, had a noticeable effect on the interaction of E2plip apo with E1p (Figure 2). Not only did many residues 
a.

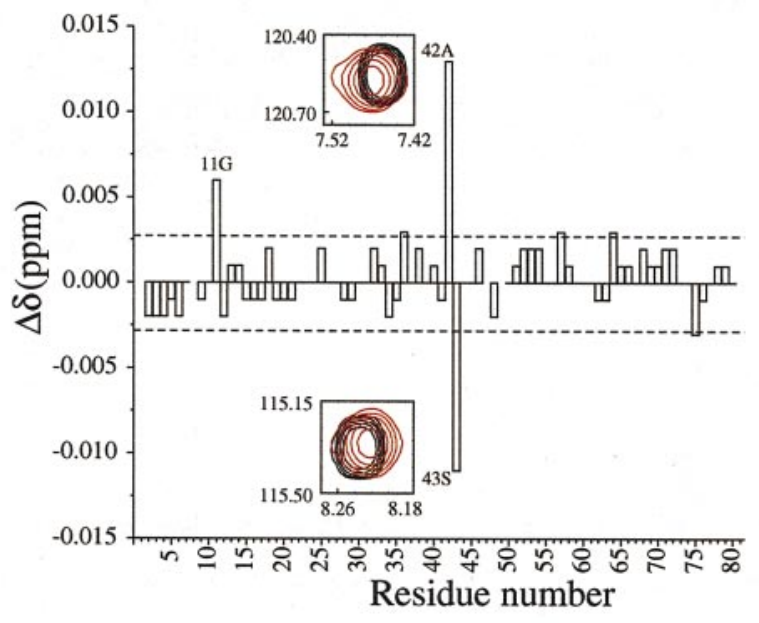

b.

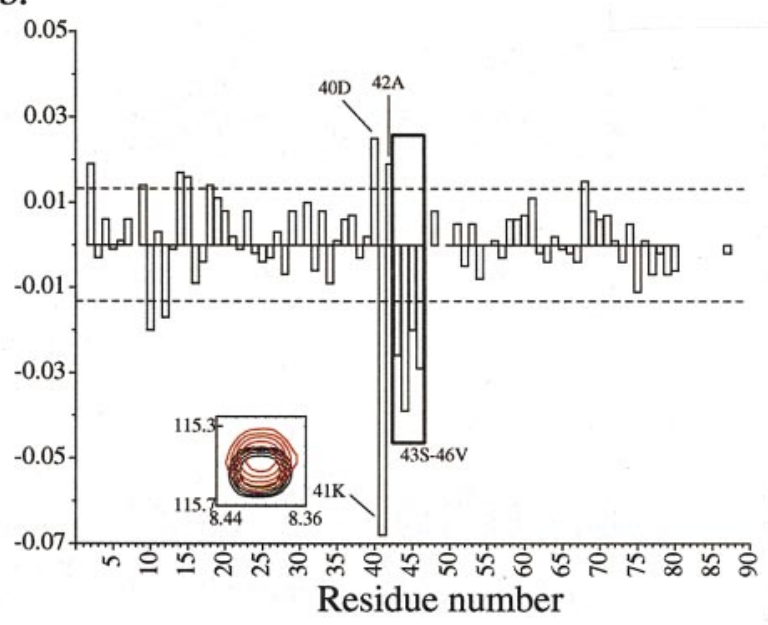

c.

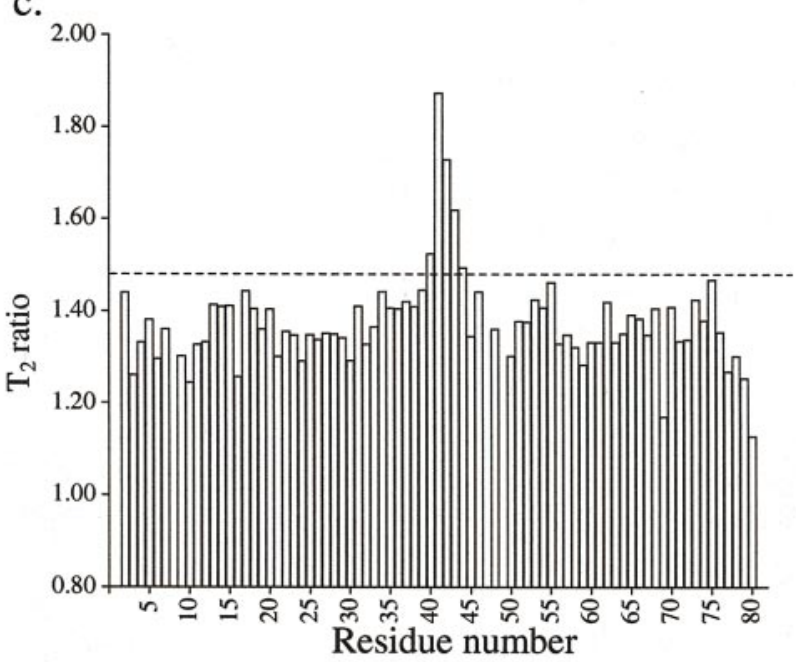

d.

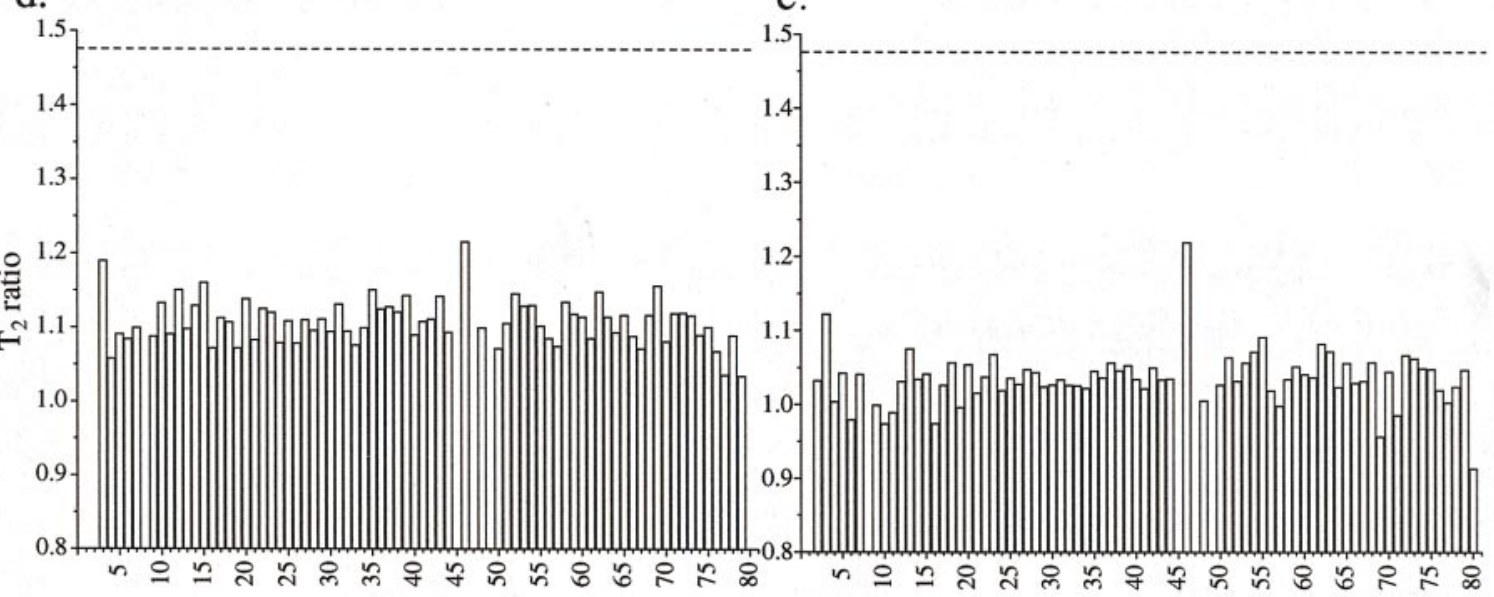

Residue number

Figure 1. The interaction of E2plipapo with E1p and E1o. (a) change in ${ }^{1} \mathrm{H}$ chemical shift and (b) change in ${ }^{15} \mathrm{~N}$ chemical shift for E2plipapo $(0.6 \mathrm{mM})$ in the presence of E1p $(0.1 \mathrm{mM}) . \Delta \delta$ was calculated by subtracting the E2plip ${ }^{\text {apo:E1p }}$ chemical shift from the free E2plipapo chemical shift. Also shown are the resonances for the E2plip apo (black) and E2plipapo:E1p (red) for residues Lys41, Ala42 and Ser43. The effects of (c) E1p, (d) E1o and (e) BSA on the $T_{2}$ times of the E2lipapo domain are also indicated. The $T_{2}$ ratio was calculated (from equation (2)) as described in Materials and Methods. The broken lines represent the threshold values calculated as described in Materials and Methods. 


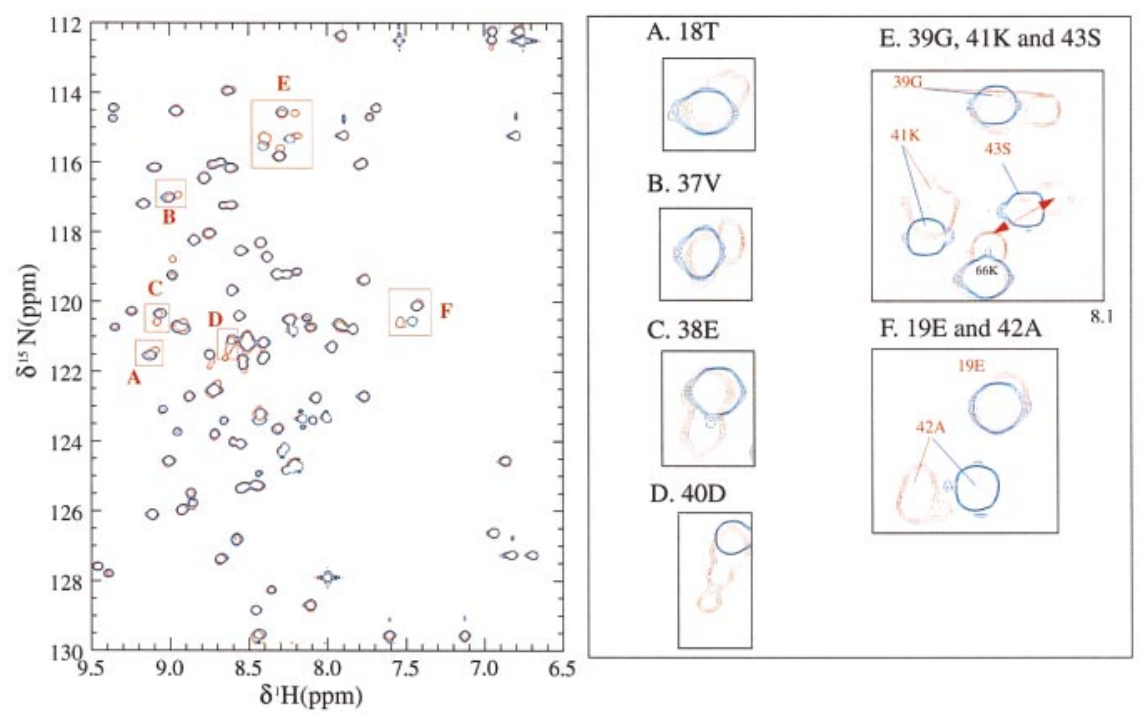

Figure 2. The effect of pyruvate on the interaction of E2plip apo with E1p. The HSQC spectrum in blue represents that of the free E2plip apo domain and the spectrum in red represents that recorded in the presence of E1p and pyruvate. The right-hand panel magnifies several cross-peaks indicated in the left-hand panel. For further details, see the text.

undergo a large change in chemical shift, far greater than seen in the presence of E1p alone, but many residues also displayed multiple resonances. Residues Thr18, Val37, Glu38, Gly39, Asp40, Lys41, Ala42 and Ser43 showed the highest degree of multiplicity (Figure 2). This could be explained by a conformational change in the lipoyl domain taking place on binding to Elp in the presence of substrate, with the conformers in slow exchange. Alternatively, and probably more likely, a conformational change in Elp itself might be responsible. On the addition of more E1p (final concentration $0.2 \mathrm{mM}$ ), the intensities of all the peaks diminished, indicating that further line broadening had occurred (data not shown). If the E1p-E2plip apo complex was in slow exchange with E1p, increasing the E1p concentration would reduce the time the domain spends in its free state, resulting in the observed increase in line broadening.

With respect to the ${ }^{1} \mathrm{H}$ nucleus, Thr18, Glu31, Val37, Gly39, Ala42 and Ser43 exhibited a significant $\Delta \delta$ (Figure 3(a)); and with respect to the ${ }^{15} \mathrm{~N}$ nucleus, Thr18, Val28, Glu38, Asp40, Lys41, Ser43, Met44, Val46, Ala48 and Lys58 showed a significant $\Delta \delta$ (Figure 3(b)). Most of these residues undergoing significant changes in chemical shift are centred around the lipoyl-lysine $\beta$-turn, except for Thr18, Val28, Glu31, Ala48 and Lys58.

The E2plip ${ }^{\text {apo }}$ domain was found to exhibit no changes in chemical shift in the presence of pyruvate alone. Likewise, E2plip ${ }^{\text {apo }}$ in the presence of pyruvate and Elo showed no significant changes in chemical shift (data not shown). The addition of 2-oxoglutarate, the natural substrate for E1o, caused a slight change in chemical shift for three residues (Gly12, Glu16, Ala29), but the changes were much smaller than those observed in the presence of Elp and pyruvate (Figure 3) and the multiple resonances for specific residues (Figure 2) were not observed. Thus, the presence of 2-oxoglutarate may promote an interaction of E2plip ${ }^{\text {apo }}$ with E1o, but any such interaction is very weak and is certainly non-productive catalytically (Graham et al., 1989; Jones et al., 2000b).

As $T_{2}$ values are calculated using resonance intensity, the presence of multiple cross-peaks for particular residues in the presence of Elp and pyruvate will influence their $T_{2}$ values. For those that could be measured, some of the $T_{2}$ values remained close in value (e.g. for Thr18, Glu38), but those of Val37 (109 ms and $87 \mathrm{~ms})$, Gly39 (112 ms and $55 \mathrm{~ms}$ ), Asp40 (81 $\mathrm{ms}$ and $109 \mathrm{~ms}$ ) and Ser43 (127 $\mathrm{ms}$ and $70 \mathrm{~ms}$ ) differed depending on which resonance intensity was used to calculate the $T_{2}$. Nevertheless, the $T_{2}$ values for E2plip ${ }^{\text {apo }}$ calculated over the whole backbone were generally lower in the presence of Elp and pyruvate, indicating that the substrate was promoting an interaction (Figure 4(a)). As Figure 4 shows, the residues most affected could not be easily identified as the trend does not appear to be smooth; but Leu34, Asp40, Lys41, Ala42, Ser43 and Met48 appeared to undergo a larger change than the majority. Although Val7 and Ala48 are above the set threshold, in the HSQC spectrum of E2plip ${ }^{\text {apo }}$ alone, the cross-peaks overlay, thereby making the calculated $T_{2}$ inaccurate, and so these changes were ignored.

In the presence of both E1o and 2-oxoglutarate, the E2plip ${ }^{\text {apo }}$ domain exhibited no significant changes in $T_{2}$ (Figure 4), as noted previously with E1o alone (Figure 1). This again would suggest that there is no interaction between E2plip ${ }^{\text {apo }}$ and E1o. 


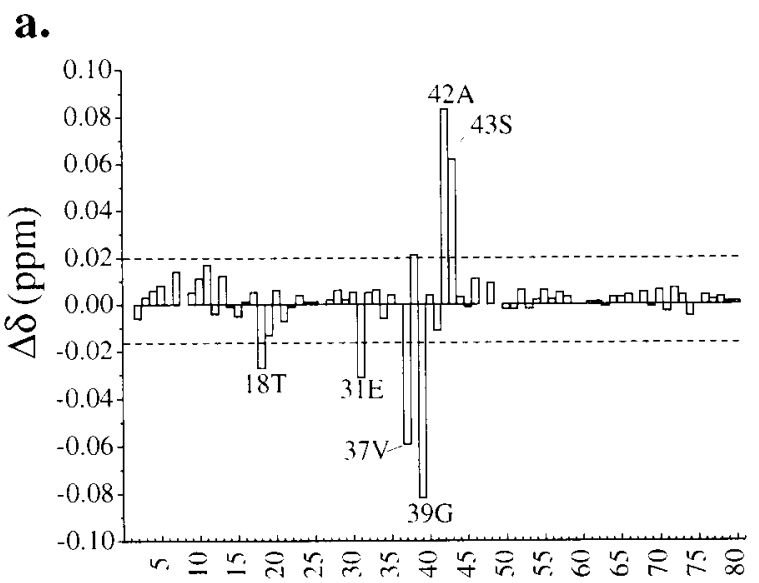

b.

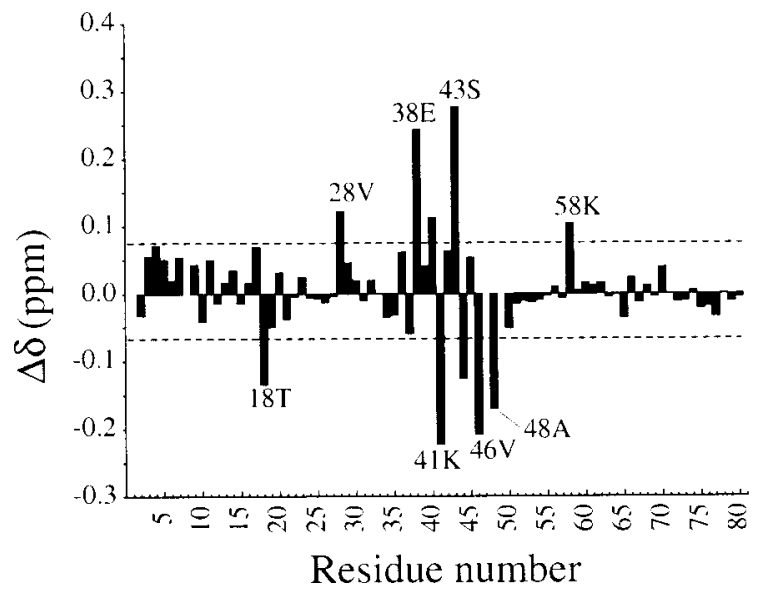

Figure 3. The effect of pyruvate on the interaction of E2plip apo with E1p. The changes in backbone (a) ${ }^{1} \mathrm{H}^{\mathrm{N}}$ and (b) ${ }^{15} \mathrm{~N}$ chemical shift are shown. $\Delta \delta$ was calculated

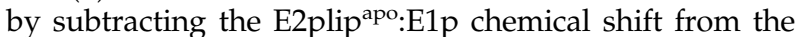
free E2plip ${ }^{\text {apo }}$ chemical shift. The broken lines represent the threshold values calculated as described in Materials and Methods. For further details, see the text.

\section{The interaction of E2plip ${ }^{\text {holo }}$ with E1p}

In the intact complex, the lipoyl domain has to be lipoylated to participate in catalysis and the natural substrate for reductive acetylation by E1p is the E2plip ${ }^{\text {holo }}$ domain. Lipoylation causes significant changes in chemical shift and the backbone dynamics of residues in the lipoyl-lysine $\beta$-turn, implying some potentially important interactions between the domain and the lipoyl-lysine sidechain (Jones et al., 2000a).

In the presence of Elp, the changes observed in chemical shift were greater for the E2plipholo (Figure 5) than for the E2plip ${ }^{\text {apo }}$ (Figure 1) domain. The largest changes were observed for residues in the lipoyl-lysine $\beta$-turn region, most notably Asp40, Lys41, Ala42, Ser43, Met44, Val46 and, to a lesser extent, Thr36 and Glu38. In addition, residues in the surface loop connecting $\beta$-strands 1 and 2 (Ile10, Gly11, Gly12 and Glu14) also under- went small yet significant changes in chemical shift. This is not entirely surprising, as the surface loop is close in space to the lipoyl-lysine $\beta$-turn and has been implicated as a contact region with Elp in previous studies (Wallis et al., 1996; Jones et al., 2000b). The chemical shifts of residues in and surrounding $\beta$-strand 7 (Gly62, Lys64 and Gly68) were also slightly perturbed. $\beta$-strand 7 lies parallel with $\beta$-strand 2 and the surface loop mentioned above. Also visible is the lipoyl-lysine amide proton (Lys41 ; Lys- $(\mathrm{H})^{15} \mathrm{~N}-\mathrm{C}(\mathrm{O})$-lip). The change in chemical shift was not as large as expected: 0.001 and $0.03 \mathrm{ppm}$ for ${ }^{1} \mathrm{H}$ and ${ }^{15} \mathrm{~N}$, respectively, compared with 0.005 and $0.08 \mathrm{ppm}$ for the Lys41 backbone amide.

The $T_{2}$ values for the E2plipholo domain in the presence of E1p generally decreased in the presence of E1p (Figure 6). The overall decrease was greater than for the E2plip apo domain, where the majority of $T_{2}$ ratios ranged between 1.2 and 1.4 (Figure 1), compared with 1.6 to 1.8 for E2plipholo. This would indicate that the interaction with Elp is significantly stronger when the domain is lipoylated. Only six residues exhibited a change in $T_{2}$ above the calculated threshold: Glu16, Lys41, and to a greater extent, Gly39, Asp40, Ala42 and Ser43. All these residues lie in the lipoyl-lysine $\beta$-turn region, apart from Glu16 which is at the $\mathrm{N}$-terminal end of $\beta$-strand 2 , close in space to the lipoyl-lysine $\beta$-turn. Although the ratio suggests that the $T_{2}$ of Lys $41^{\zeta}$ has not changed significantly, the absolute change is quite high ( $376 \mathrm{~ms}$ for the free E2plip ${ }^{\text {holo }}$ and $226 \mathrm{~ms}$ in the presence of E1p i.e. $\Delta T_{2}=150 \mathrm{~ms}$ ).

\section{The interaction of E2plip ${ }^{\text {holo }}$ with E10 and BSA}

The lipoyl group is an essential part of a functional lipoyl domain. Contrary to what was observed for E2plip ${ }^{\text {apo }}$, the E2plip ${ }^{\text {holo }}$ domain does appear to interact weakly with E1o. Thus, small but significant changes in chemical shift were observed in the presence of E1o (Figure 5), the most notable involving Ala42 and also Lys41 ${ }^{\zeta}$ (not shown). To confirm the significance of these changes, the same experiments were run with BSA replacing Elo; apart from the $\Delta \delta$ in the ${ }^{15} \mathrm{~N}$ dimension for Ser43, all the other changes were judged to be significant.

There is a general drop in $T_{2}$ values, by a factor of ca 1.5, for the E2plip ${ }^{\text {holo }}$ domain in the presence of E1o (Figure 6). This is significantly greater than that observed for E2lipapo in the presence of E1o (Figure 1(d)). No one residue appears to be affected to any extent greater than that of the global change, suggesting that the interaction with Elo may be restricted to the lipoyl group. The $T_{2}$ ratio of Lys $41^{\zeta}$ indicates that although it is the closest bonded amide to the dithiolane ring of the lipoyl group, it does not undergo any significant change. Nonetheless, the absolute change $\left(\Delta T_{2}=139 \mathrm{~ms}\right)$ is quite high. 

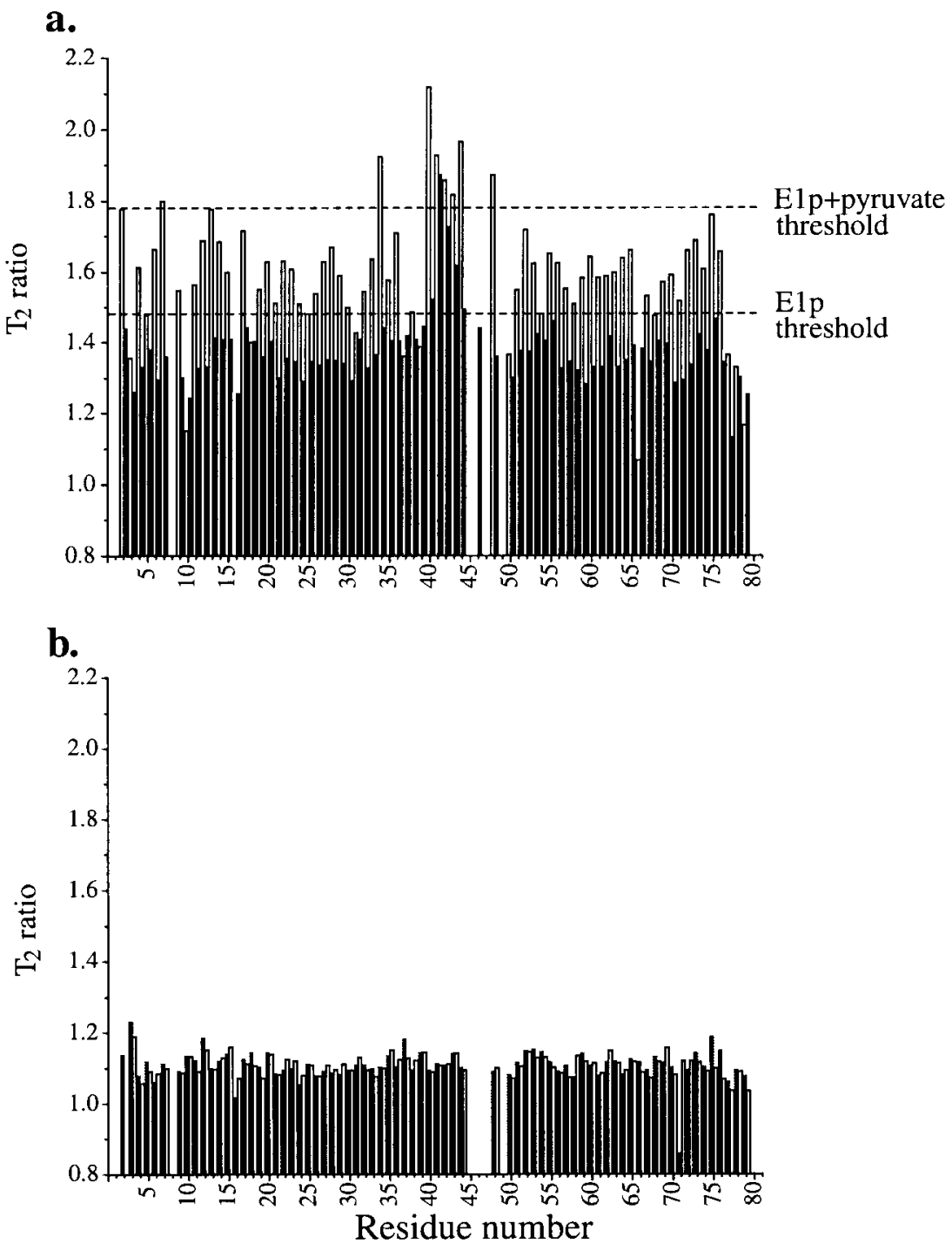

Figure 4. The effect of 2-oxo acid substrate on the $T_{2}$ times of E2plip ${ }^{\text {apo }}$ in the presence of Elp or Elo. (a) Comparison of $T_{2}$ ratios in the presence of E1p alone (filled) and E1p plus pyruvate (open). The broken lines are the threshold values calculated as described in Materials and Methods. (b) Comparison of the $T_{2}$ ratios of in the presence of E1o (open bars) or E1o plus 2-oxoglutarate (filled bars). For further details, see the text.

To test whether the $T_{2}$ data obtained for E2plip ${ }^{\text {holo }}$ in the presence of E1o were attributable to a specific interaction, BSA was again used in place of Elo. No significant interaction between E2lip ${ }^{\text {holo }}$ and BSA could be detected, as judged by the lack of change in the $T_{2}$ values.

\section{Mapping the interaction sites on the surface of E2plip}

For the E2plip ${ }^{\text {apo }}$ domain, both the chemical shift and $T_{2}$ data suggest that the main region undergoing an interaction with E1p is the lipoyl-lysine $\beta$-turn region and residues predominantly in the succceeding $\beta$-strand 5 (Figure 7 ). The other residue thought to be involved in the interaction is Gly11, which lies close in space to the lipoyl-lysine $\beta$-turn in the surface loop linking $\beta$-strands 1 and 2. Neither E1o nor BSA showed any evidence of an interaction with E2plip ${ }^{\text {apo }}$.

In the presence of E1p and pyruvate, the interaction also appeared to be centred on the lipoyl- lysine region, with residues in both $\beta$-strands 4 and 5 of E2plip apo now involved. The chemical shift data also identify a small patch of residues that may participate in the interaction: Ala48, Val28 and Glu31. All these residues lie close in space to each other (Figure 7). The chemical shift data also imply that Thr18, another nearby residue in $\beta$-sheet 2 , and Lys58, on the opposite face of the protein, may interact with E1p. The $T_{2}$ data additionally indicate Asp13 in the surface loop as undergoing an interaction (Figure 7).

The interaction of the E2plip ${ }^{\text {holo }}$ domain with E1p is focused on the half of the protein containing the lipoyl-lysine residue (Figure 8). Residues in the lipoyl-lysine $\beta$-turn and its constituent $\beta$-strands 4 and 5 , together with residues in the nearby surface loop, in $\beta$-strand 2 and, to a lesser extent, in and adjacent to $\beta$-strand 7 , all undergo a change in chemical shift. The $T_{2}$ data mirror the chemical shifts, except that the interactions appear to be restricted to the lipoyl-lysine $\beta$-turn region and Glu16 in $\beta$-strand 2. 

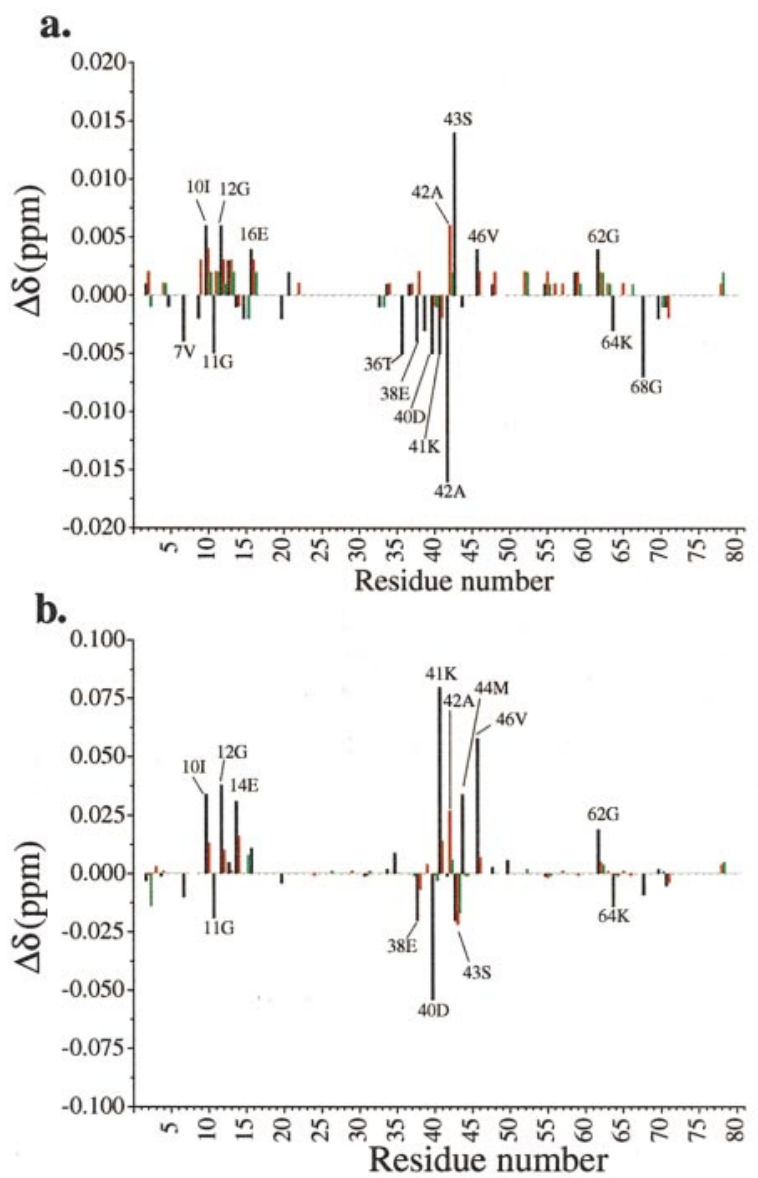

Figure 5. The effect of Elp, Elo and BSA on the chemi-

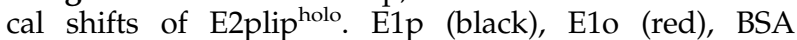
(green). Shown above is the change in (a) ${ }^{1} \mathrm{H}$ and (b) ${ }^{15} \mathrm{~N}$ chemical shift. $\Delta \delta$ was calculated by subtracting the E2plipholo:E1p chemical shift from the free E2plipholo chemical shift. For further details, see the text.

\section{Discussion}

Previous NMR studies to examine the interaction of the apo-lipoyl domain of B. stearothermophilus E2p with its cognate $\alpha_{2} \beta_{2}$ E1p indicated various regions of the domain as being involved (Wallis et al., 1996; Howard et al., 2000). The surface loop linking $\beta$-strands 1 and 2 (Figure 7) was thought to be of particular importance, but there was no indication that the lysine residue that would carry the lipoyl group (Lys41 in the B. stearothermophilus domain) underwent an interaction with E1p. These experiments did not involve the lipoylated form of the domain, the natural substrate of E1, as the lipoylated domain appeared to bind too tightly to the $\alpha_{2} \beta_{2}$ E1p, causing extensive line broadening (Wallis et al., 1996).

Here, the E. coli E1p has the $\alpha_{2}$ quaternary structure. In this instance, our results indicate that the lipoyl-lysine and adjacent residues in the lipoyl domain are consistently involved in the interaction with E1p. The prominent surface loop linking
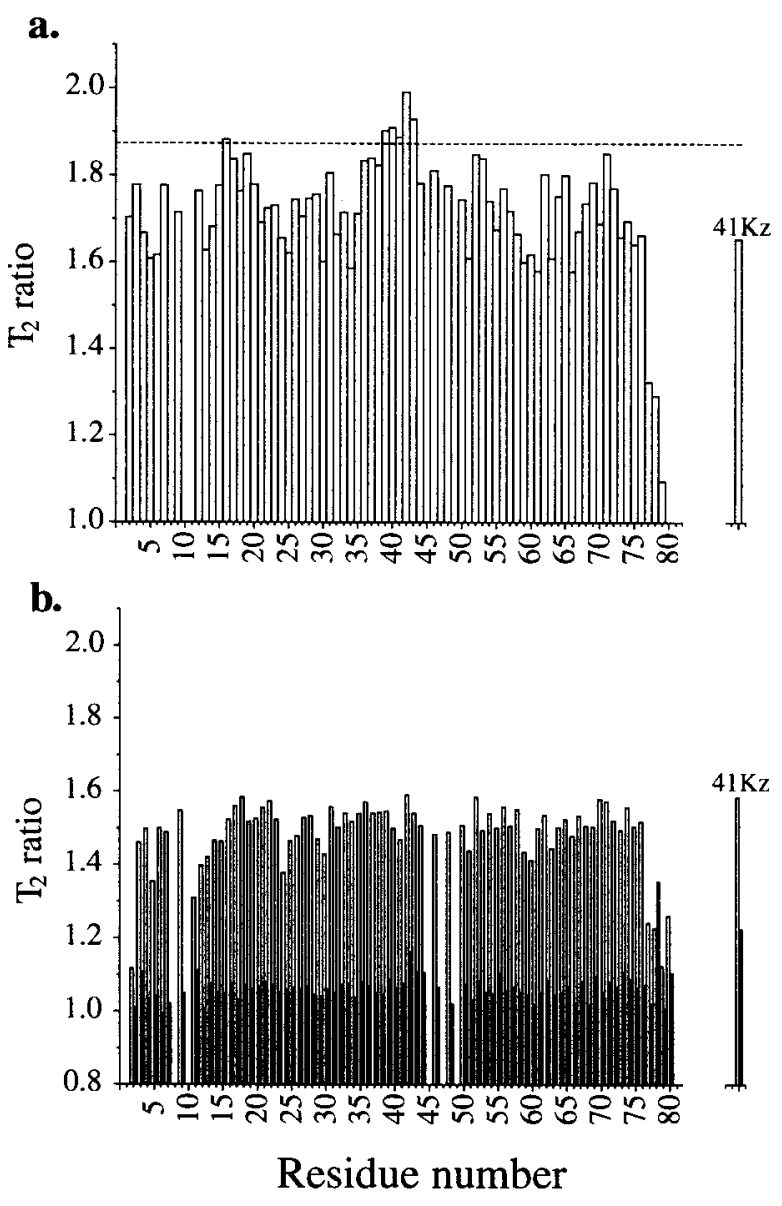

Figure 6. The effect of the interaction of E1p, E1o and BSA on the E2plip ${ }^{\text {holo }} T_{2}$ times. (a) E1p; (b) Elo (unfilled) and BSA (black). The $T_{2}$ ratio was calculated (from equation (2)) as described in Materials and Methods. The broken line represents the threshold value calculated as described in Materials and Methods. For further details, see the text.

$\beta$-strands 1 and 2 also seems to have a role to play, as it does in other complexes (Wallis et al., 1996; Berg et al., 1998; Jones et al., 2000b). However, in the E. coli E2plip ${ }^{\text {apo }}$ domain, only Gly11 in the loop undergoes what was classed as a significant change in chemical shift, and only the lipoyl-lysine region undergoes a $T_{2}$ change (Figure 1 ). Nonetheless, the overall backbone $T_{2}$ times drop from $147 \mathrm{~ms}$ to $102 \mathrm{~ms}$ in the presence of E1p, indicating that an E2plip ${ }^{\text {apo }}$-E1p complex has transiently formed. From equation (1), the lifetime of the E2plip ${ }^{\text {apo }}$ spent in the uncomplexed state $\left(t_{\text {lip }}\right)$ was estimated at about $375 \mathrm{~ms}$. In contrast, in the presence of Elo or BSA, the average $T_{2}$ values for the structured region of the lipoyl domain remain close to the values for the free E2plip ${ }^{\text {apo }}$, at about $132 \mathrm{~ms}$ and $140 \mathrm{~ms}$, respectively.

The most notable effect on the E2plip ${ }^{\text {apo-E1p }}$ interaction was that observed on adding the substrate, pyruvate (Figure 2). Decarboxylation of 


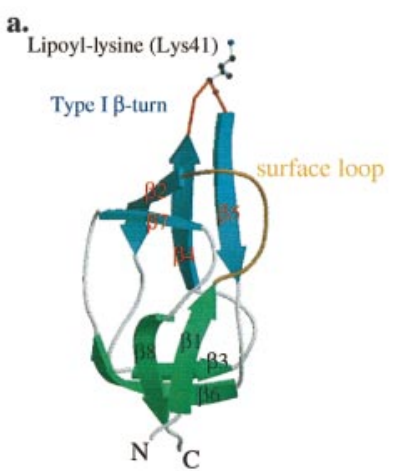

b.
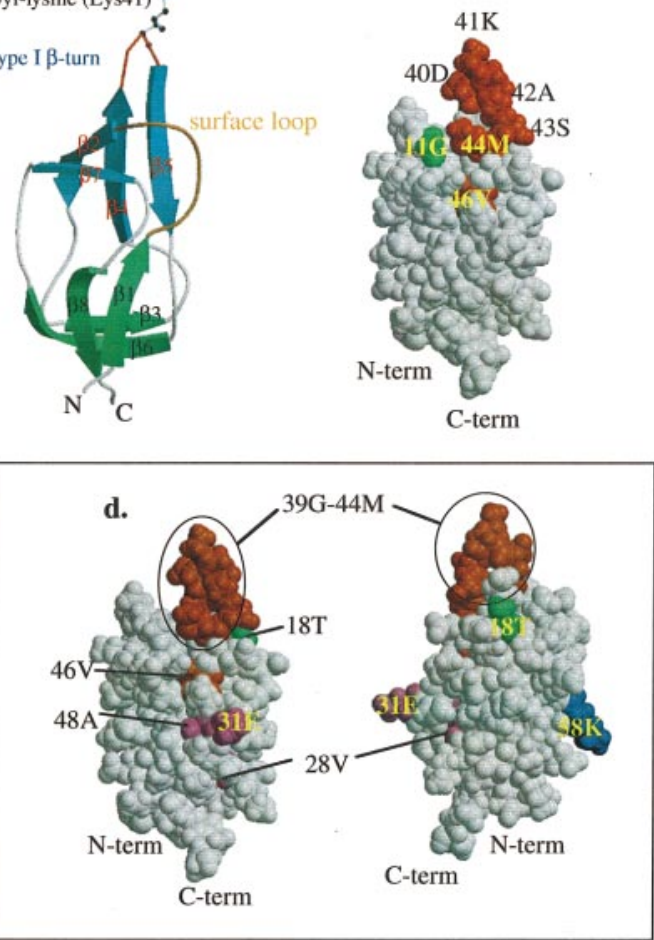

pyruvate by E1p would lead to the formation of 2-(1-hydroxyethylidene)-ThDP and this, presumably through an effect on E1p structure, caused not only major changes in chemical shift for E2plip ${ }^{\text {apo }}$, but also in the $T_{2}$ values (Figures 3 and 4). The crystal structure of an $\alpha_{2} \beta_{2}$ E1p from P. putida shows that the active (thiamin diphosphate-binding) site is well buried at the bottom of a $20 \AA$ funnel-shaped cavity (Ævarsson et al., 1999). Formation of 2-(1-hydroxyethylidene)-ThDP may c.
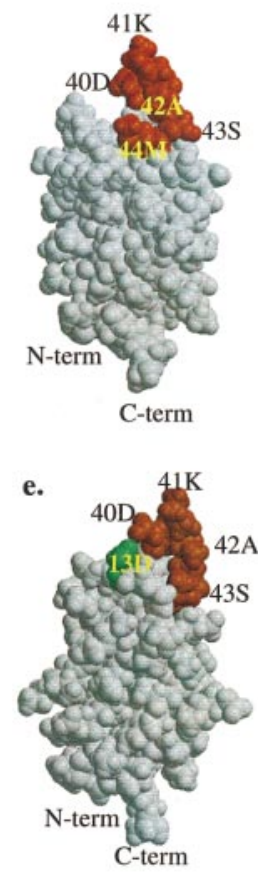

Figure 7. Points of contact between E2plip ${ }^{\text {apo }}$ and E1p. (a) Schematic representation of the E2plip apo domain structure determined previously (Jones et al., 2000a). Each $\beta$-strand is labelled, together with a prominent surface loop (orange) and the lipoyl-lysine (Lys41) $\beta$-turn (red). Residues exhibiting significant changes in (b) chemical shift and (c) $T_{2}$ values, in the presence of E1p. The effect of pyruvate on the (d) chemical shift and (e) $T_{2}$ values of E2plip apo in the presence of E1p. The two orientations shown in (d) are related by a $90^{\circ}$ rotation about the $z$ axis. The diagram was produced using MOLSCRIPT (Kraulis, 1991). induce a conformational change in Elp affecting access to this unusual active site.

In general, the measured $T_{2}$ values for the

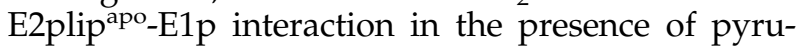
vate were lower than in the absence of pyruvate, the average $T_{2}$ over the backbone being $88 \mathrm{~ms}$. The E2plip ${ }^{\text {apo }}$ domain is evidently binding more tightly and spending less time in the free state, with $t_{\text {lip }}$ estimated at $237 \mathrm{~ms}$. The analysis of chemical shifts implicated a small cluster of residues, Val28, Glu31

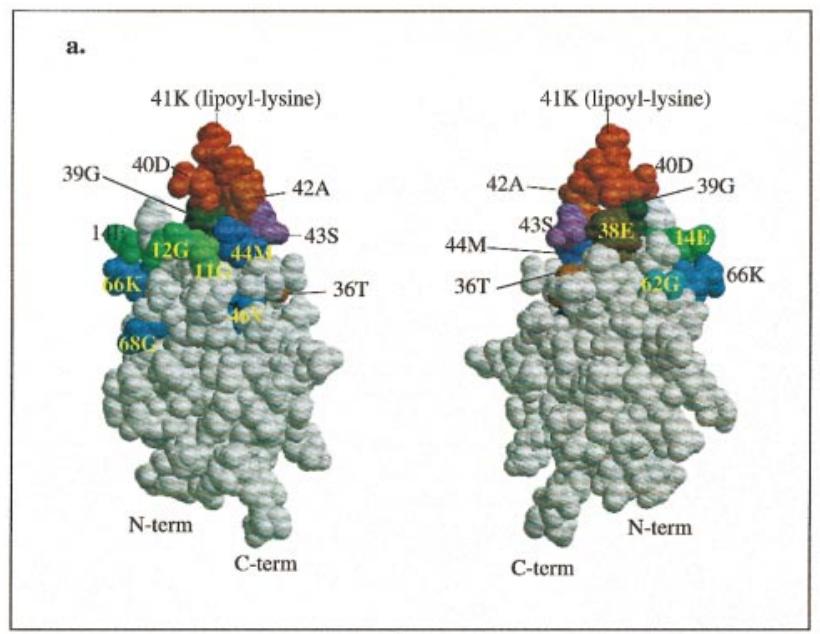

b.

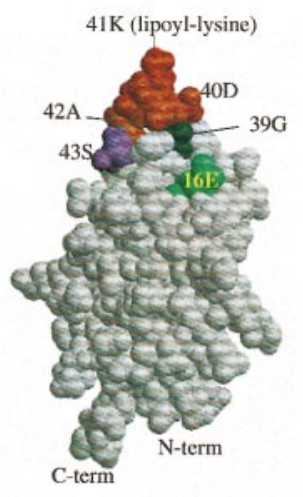

Figure 8. Points of contact between E2plip ${ }^{\text {holo }}$ and E1p. Residues exhibiting significant changes in (a) chemical shift and (b) $T_{2}$ values, in the presence of E1p. The two orientations shown in (a) are related by a $180^{\circ}$ rotation about the $z$ axis. The diagram was produced using MOLSCRIPT (Kraulis, 1991). 
and Ala48, as being involved in the interaction with Elp in the presence of substrate, with the side-chain of Glu31 protruding out from the structure.

The problem with the chemical shift data, which in turn affects the calculation of the $T_{2}$ times, is the appearance of multiple cross-peaks on the addition of pyruvate (Figure 2). The reason for this is not known but multiple cross-peaks have also been observed when the lipoylated domain (E2plip ${ }^{\text {holo }}$ ) is reductively acetylated (Jones et al., 2000a). One possible explanation is that the E2plip ${ }^{\text {apo }}$ domain undergoes a conformational change on binding to the substrate-loaded E1p and that this "new" conformation is in slow exchange with one or more other conformations. It is unlikely that one is observing the E1p-bound form of E2plip ${ }^{\text {apo }}$ in slow exchange with free E2plip ${ }^{\text {apo }}$, as the size of the

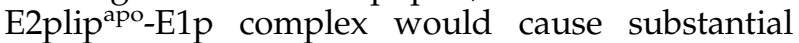
line broadening. Another, more likely, explanation could be different conformations of the two subunits in E1p in the presence of ThDP and pyruvate (Hennig et al., 1997); if the two subunits of the Elp $\alpha_{2}$ dimer have slightly different conformations, the multiple cross-peaks could be the result of E2plip ${ }^{\mathrm{a}-}$ po interacting with either one or other of the two active sites.

The number of residues undergoing a significant change in chemical shift in the presence of Elp was found to have increased for the E2plip ${ }^{\text {holo }}$ domain compared with E2plip ${ }^{\text {apo }}$, and to be restricted to the lipoyl-lysine-containing half of the domain (Figures 7 and 8). An interesting feature of some of these residues, most notably Ile10, Gly12, Glu14, Thr36, Gly62 and Gly68, is that they were not affected by the presence or absence of pyruvate in the interaction of the E2plip ${ }^{\text {apo }}$ domain (Figures 1 and 3). What they have in common is that they also undergo a significant change in chemical shift upon lipoylation of the domain (Jones et al., 2000a). Thus, the chemical shift changes observed for these residues in the presence of E1p may simply reflect an interaction of the lipoyl group with E1p.

The lipoyl-lysine region is the only part of the E2p $\mathrm{p}^{\text {holo }}$ domain that showed significant changes in both $T_{2}$ and chemical shift (Figure 8). The $T_{2}$ ratios for E2plip ${ }^{\text {holo }}$ had a larger background fluctuation, making interpretation difficult. Only six residues, Glu16, Gly39, Asp40, Lys41, Ala42 and Ser43, underwent a major change, although Lys $41^{\zeta}$ did undergo a large absolute change $\left(\Delta T_{2}=150 \mathrm{~ms}\right)$. However, in the presence of E1p, the average $T_{2}$ values across the backbone were lower for E2pli$\mathrm{p}^{\text {holo }}$ than E2plip ${ }^{\text {apo }}(82.0 \mathrm{~ms}$ and $102 \mathrm{~ms}$, respectively). This implies that the E2plip-E1p interaction is tighter when the domain is lipoylated, as noted previously in studies of the B. stearothermophilus $\alpha_{2} \beta_{2}$ E1p-E2plip interaction (Wallis et al., 1996).

The results obtained for the interaction of E1o with E2plip ${ }^{\text {holo }}$ indicate that there is a small contribution to the recognition process from the lipoyl group itself. Thus, there were slight yet significant changes in chemical shift for E2plip ${ }^{\text {holo }}$ in the pre- sence of E1o (Figure 5), involving residues mainly in the lipoyl-lysine $\beta$-turn region. The general decrease in the $T_{2}$ times, with an average of $94 \mathrm{~ms}$, well below that observed (132 ms) for E2plip ${ }^{\text {apo }}$ in the presence of E1o (Figure 1) would imply the formation of a transient complex. The lifetime of the unbound state decreases from ca $2310 \mathrm{~ms}$ for E2plip apo to ca $286 \mathrm{~ms}$ for E2plip holo. However, given the lack of fluctuation in the background $T_{2}$ ratio (Figure 6), it would appear that the interaction is non-specific with respect to the protein. The most likely explanation is that the lipoyl-lysine residue but not the domain is recognised by E1o, though not in a catalytically productive way such that reductive acetylation can follow (Graham et al., 1989; Jones et al., 2000b). It is unfortunate that experiments with Elp in the presence of pyruvate could not be performed as catalysis would occur, resulting in reductive acetylation of E2plip ${ }^{\text {holo }}$.

In general, it is now clear that the El components of 2-oxo acid dehydrogenase complexes can recognize the lipoyl group as such, but that specific recognition of the lipoyl domain to which it is attached is required for reductive acylation to ensue. The surface loop on the domain between $\beta$-strands 1 and 2, close in space to the lipoyl-lysine $\beta$-turn, is an important transient contact point between $\mathrm{El}$ and the lipoyl domain. However, a mosaic of other contacts distributed chiefly over the half of the domain that contains the lipoyllysine residue is required to ensure a productive encounter. The structural subtleties involved can now perhaps be further defined by reference to the structure of the El component (Ævarsson et al., 1999) and the pattern of interaction sites on the lipoyl domain disclosed by the NMR experiments, as above.

\section{Materials and Methods}

\section{Sample preparation}

The ${ }^{15} \mathrm{~N}$-labelled E2plip ${ }^{\text {apo }}$ domain was purified from over-expressing E. coli cells as described elsewhere (Dardel et al., 1991; Ricaud et al., 1996; Jones et al., 2000a). The E2plipholo domain was generated in vitro using LplA, ATP-Mg and R-lipoate (Asta Pharmaceuticals) and its identity confirmed by positive-ion electrospray mass spectrometry, all as described elsewhere (Jones et al., 2000b). The domains were exchanged into $20 \mathrm{mM}$ sodium phosphate buffer ( $\mathrm{pH}$ 6.85).

Batches of 11 of LB medium containing $100 \mu \mathrm{g} / \mathrm{ml}$ ampicillin were inoculated with a single colony of $E$. coli BL21(DE3) cells freshly transformed with the plasmid pETE1p, which carries a gene encoding the pyruvate decarboxylase (E1p) of the PDH complex from E. coli (P.A.R. \& R.N.P., unpublished work). The cells were grown at $37^{\circ} \mathrm{C}$ until an $A_{600}$ of $0.6-0.8$ was reached, and protein expression was induced with IPTG (final concentration of $0.6 \mathrm{mM}$ ) at $25^{\circ} \mathrm{C}$ for 16 hours. Cells were harvested by centrifugation at $5000 \mathrm{~g}$ for ten minutes, resuspended $(10 \mathrm{ml} / \mathrm{g}$ of wet cell pellet) in buffer $\mathrm{A}$ (20 mM sodium phosphate, $0.5 \mathrm{mM}$ EDTA, $0.1 \mathrm{mM}$ DTT, pH 7.0) containing $1 \mathrm{mM}$ PMSF, disrupted in a French press (SLM-AMINCO), and the cell debris 
removed by centrifugation $(15,000 \mathrm{~g}$ for one hour at $4{ }^{\circ} \mathrm{C}$ ). Recombinant E1p was fractionally precipitated from the cell-free extract with ammonium sulphate (20$60 \%$ saturation), the precipitate was dissolved in buffer B (20 mM sodium phosphate, $0.5 \mathrm{mM}$ EDTA, $0.1 \mathrm{mM}$ DTT, $1 \mathrm{M}\left(\mathrm{NH}_{4}\right)_{2} \mathrm{SO}_{4}, \mathrm{pH}$ 7.0) (3 ml per mg of precipitated protein) and applied to a HiLoad ${ }^{(i}$ 16/10 Phenyl Sepharose column HP (Pharmacia), previously equilibrated with buffer B. Recombinant E1p was eluted from the column by applying a linear gradient from buffer B to buffer A $(200 \mathrm{ml})$ at a flow rate of $2.5 \mathrm{ml} / \mathrm{min}$. Fractions containing E1p were pooled, dialysed against buffer C $(20 \mathrm{mM}$ Tris- $\mathrm{HCl}, 0.5 \mathrm{mM}$ EDTA, $0.1 \mathrm{mM}$ DTT, $\mathrm{pH} 7.0$ ) and concentrated to $15 \mathrm{ml}$ using a $50 \mathrm{kDa}$ cut-off Ultrafree $^{\mathbb{R}}-15$ concentrator (Millipore) before being loaded onto a HiLoad ${ }^{\circledR} \mathrm{Q}$ HR 16/10 column, previously equilibrated with buffer $\mathrm{C}$. E1p was then eluted from the column by applying a linear gradient $(120 \mathrm{ml})$ of $0-0.5 \mathrm{M}$ $\mathrm{NaCl}$ in buffer $\mathrm{C}$ at a flow rate of $2.5 \mathrm{ml} /$ minute. Fractions of interest were pooled, dialysed against $20 \mathrm{mM}$ sodium phosphate buffer ( $\mathrm{pH} 6.0)$, concentrated and stored at $-80^{\circ} \mathrm{C}$.

\section{Interaction studies}

All samples contained the ${ }^{15} \mathrm{~N}$-labelled lipoyl domain at a concentration of $0.6 \mathrm{mM}, \mathrm{ThDP}-\mathrm{Mg}$ at a molar ratio (E1:ThDP-Mg) of $1: 2,0.02 \%(\mathrm{w} / \mathrm{v})$ sodium azide, $10 \%$ ${ }^{2} \mathrm{H}_{2} \mathrm{O}$ and $18 \mathrm{mM}$ sodium phosphate $(\mathrm{pH}$ 6.85). For the studies of the E2plip ${ }^{\text {apo }}$ interaction, E1p was present at a concentration of $0.1 \mathrm{mM}$ or $0.2 \mathrm{mM}, \mathrm{E} 1 \mathrm{o}$ at a concentration of $0.1 \mathrm{mM}$, and bovine serum albumin (BSA) at a concentration of $0.1 \mathrm{mM}$, as appropriate. Where applicable, the 2-oxo acid was added at a molar ratio (2-oxo acid:E1) of 2:1 and left at room temperature (circa $22{ }^{\circ} \mathrm{C}$ ) for 30 minutes to allow oxidative decarboxylation to occur. For the E2plipholo domain, E1p, E1o or BSA were present at a concentration of $0.1 \mathrm{mM}$. No 2-oxo acid was added. The total volume for all samples was $600 \mu \mathrm{l}$ and the same buffer was used throughout.

\section{NMR spectroscopy}

\section{Chemical shift analysis}

Heteronuclear single quantum correlation (HSQC) spectra were recorded using 16 scans for each $t_{1}$ point and 256 increments at $298 \mathrm{~K}$, with spectral widths of $6009.625 \mathrm{~Hz}\left({ }^{1} \mathrm{H}\right)$ and $927.29 \mathrm{~Hz}\left({ }^{15} \mathrm{~N}\right)$. The assignment of the spectra has been described (Jones et al., 2000a). The chemical shifts were assigned using the program ANSIG3.3 (Kraulis, 1989).

\section{$T_{2}$ measurements}

The ${ }^{15} \mathrm{~N} T_{2}$ values were obtained as described by Jones et al. (2000a); ${ }^{15} \mathrm{~N} \mathrm{~T}_{2}$ data were obtained using delay values of 7.92, 300.96, 47.52, 142.56, 79.2, 205.92 and $15.84 \mathrm{~ms}$, recorded in that order. The spectral width in the ${ }^{1} \mathrm{H}$ and ${ }^{15} \mathrm{~N}$ dimensions were $10000 \mathrm{~Hz}$ and $1672.17 \mathrm{~Hz}$, respectively. $T_{2}$ times were calculated as described by Jones et al. (2000a).

The threshold levels set for a change in chemical shift or in ${ }^{15} \mathrm{~N}_{2}$ value due to secondary processes is the mean of all the chemical shift changes $(\Delta \delta)$ or $T_{2}$ ratios plus one standard deviation (Howard et al., 2000).

\section{Acknowledgements}

We thank the Biotechnology and Biological Sciences Research Council for the award of a research grant (to R.N.P.) and the BBSRC and The Wellcome Trust for support of the core facilities of the Cambridge Centre for Molecular Recognition. D.D.J is grateful to the BBSRC and AdProTech for the award of a CASE Studentship. We thank Dr Paul Ricaud for the gift of E1o and Mr C. Fuller for the preparation of LplA and skilled technical assistance. We also thank Drs R.W. Broadhurst and M.J. Howard for advice on running and analysing the NMR experiments.

\section{References}

Ævarsson, A., Seger, K., Turley, S., Sokatch, J. R. \& Hol, W. G. J. (1999). Crystal structure of 2-oxoisovalerate and dehydrogenase and the architecture of 2-oxo acid dehydrogenase multienzyme complexes. Nature Struct. Biol. 6, 785-792.

Berg, A., de Kok, A. \& Vervoort, J. (1994). Sequential ${ }^{1} \mathrm{H}$ and ${ }^{15} \mathrm{~N}$ nuclear magnetic resonance assignments and secondary structure of the N-terminal lipoyl domain of the dihydrolipoyl transacetylase component of the pyruvate dehydrogenase complex from Azotobacter vinelandii. Eur. J. Biochem. 221, 87100.

Berg, A., Smits, O., de Kok, A. \& Vervoort, J. (1995). Sequential ${ }^{1} \mathrm{H}$ and ${ }^{15} \mathrm{~N}$ nuclear magnetic resonance assignments and secondary structure of the lipoyl domain of the 2-oxoglutarate dehydrogenase complex from Azotobacter vinelandii: evidence for high structural similarity with the lipoyl domain of the pyruvate dehydrogenase complex. Eur. J. Biochem. 234, 148-159.

Berg, A., Vervoort, J. \& de Kok, A. (1996). Solution structure of the lipoyl domain of the 2-oxoglutarate dehydrogenase complex from Azotobacter vinelandii. J. Mol. Biol. 261, 432-442.

Berg, A., Vervoort, J. \& de Kok, A. (1997). Three-dimensional structure in solution of the N-terminal lipoyl domain of the pyruvate dehydrogenase complex from Azotobacter vinelandii. Eur. J. Biochem. 244, 352360.

Berg, A., Westphal, A. H., Bosma, H. J. \& de Kok, A. (1998). Kinetics and specificity of reductive acylation of wild-type and mutated lipoyl domains of 2-oxo-acid dehydrogenase complexes from Azotobacter vinelandii. Eur. J. Biochem. 252, 45-50.

Dardel, F., Laue, E. D. \& Perham, R. N. (1991). Sequence-specific ${ }^{1} \mathrm{H}$ NMR assignments and secondary structure of the lipoyl domain of the Bacillus stearothermophilus pyruvate dehydrogenase multienzyme complex. Eur. J. Biochem. 201, 203-209.

Dardel, F., Davis, A. L., Laue, E. D. \& Perham, R. N. (1993). Three-dimensional structure of the lipoyl domain from Bacillus stearothermophilus pyruvate dehydrogenase multienzyme complex. J. Mol. Biol. 229, 1037-1048.

de Kok, A., Hengeveld, A. F., Martin, A. \& Westphal, A. H. (1998). The pyruvate dehydrogensase multienzyme complex from Gram-negative bacteria. Biochim. Biophys. Acta, 1385, 353-366.

Dwek, R. A. (1973). Nuclear Magnetic Resonance (N.M.R.) in Biochemistry, Clarendon Press, Oxford, UK. 
Graham, L. D. \& Perham, R. N. (1990). Interactions of lipoyl domains with the Elp subunits of the pyruvate dehydrogenase multienzyme complex from Escherichia coli. FEBS Letters, 262, 241-244.

Graham, L. D., Packman, L. C. \& Perham, R. N. (1989). Kinetics and specificity of reductive acylation of lipoyl domains from 2-oxo acid dehydrogenase complexes. Biochemistry, 28, 1574-1581.

Green, D. E., Morris, T. W., Green, J., Cronan, J. E. \& Guest, J. R. (1995). Purification and properties of the lipoate protein ligase of Escherichia coli. Biochem. J. 309, 853-862.

Griffin, T. A., Wynn, R. M. \& Chuang, D. T. (1990). Expression and assembly of mature apotransacylase (E2b) of bovine branched-chain alpha-keto acid dehydrogenase complex in Escherichia coli: demonstration of transacylase activity and modification by lipoylation. J. Biol. Chem. 265, 12104-12110.

Hengeveld, A. F., Westphal, A. H. \& de Kok, A. (1997). Expression and characterisation of the homodimeric E1 component of the Azotobacter vinelandii pyruvate dehydrogenase complex. Eur. J. Biochem. 250, 260268.

Hennig, J., Kern, G., Neef, H., Spinka, M., Bisswanger, H. \& Hubner, G. (1997). Molecular mechanism of regulation of the pyruvate dehydrogenase complex from E. coli. Biochemistry, 36, 15772-15779.

Howard, M. J., Fuller, C., Broadhurst, R. W., Perham, R. N., Tang, J. G., Quinn, J., Diamond, A. G. \& Yeaman, S. J. (1998). Three-dimensional structure of the major autoantigen in primary biliary cirrhosis. Gastroenterology, 115, 139-146.

Howard, M. J., Chauhan, H. J., Domingo, G. J., Fuller, C. \& Perham, R. N. (2000). Protein-protein interaction revealed by NMR $T_{2}$ relaxation experiments: the lipoyl domain and $\mathrm{El}$ component of the pyruvate dehydrogenase multienzyme complex of Bacillus stearothermophilus. J. Mol. Biol. 295, 10231037.

Jardetsky, O. \& Roberts, G. C. K. (1981). NMR in Molecular Biology, Academic Press, New York.

Jones, D. D., Stott, K. M., Howard, M. J. \& Perham, R. N. (2000a). Restricted motion of the lipoyl-lysine swinging arm in the pyruvate dehydrogenase complex of Escherichia coli. Biochemistry, 39, 8448-8459.

Jones, D. D., Horne, J. J., Reche, P. A. \& Perham, R. N. (2000b). Structural determinants of post-translational modification and catalytic specificity for the lipoyl domains of the pyruvate dehydrogenase multienzyme complex of Escherichia coli. J. Mol. Biol. 295, 289-306.

Jordan, S. W. \& Cronan, J. E. (1997a). A new metabolic link: the acyl carrier protein of lipid synthesis donates lipoic acid to the pyruvate dehydrogenase complex in Escherichia coli and mitochondria. J. Biol. Chem. 272, 17903-17906.

Jordan, S. W. \& Cronan, J. E. (1997b). Biosynthesis of lipoic acid and posttranslational modification with lipoic acid in Escherichia coli. Methods Enzymol. 279, 176-183.
Kraulis, P. J. (1989). Ansig - a program for the assignment of protein ${ }^{1} \mathrm{H}$ 2D-NMR spectra by interactive computer graphics. J. Magn. Reson. 84, 627-633.

Kraulis, P. J. (1991). MOLSCRIPT: a program to produce both detailed and schematic plots of protein structure. J. Appl. Crystallog. 24, 946-950.

Lian, L. \& Roberts, G. C. K. (1993). NMR of Macromolecules, IRL Press, Oxford, UK.

Macherel, D., Bourguignon, J., Forest, E., Faure, M., Cohen, Addad C. \& Douce, R. (1996). Expression, lipoylation and structure determination of recombinant pea H-protein in Escherichia coli. Eur. J. Biochem. 236, 27-33.

Matsuo, H., Walters, K. J., Teruya, K., Tanaka, T., Gassner, G. T., Lippard, S. J., Kyogoku, Y. \& Wagner, G. (1999). Identification by NMR spectroscopy of residues at contact surfaces in large, slowly exchanging macromolecular complexes. J. Am. Chem. Soc. 121, 9903-9904.

Morris, T. W., Reed, K. E. \& Cronan, J. E. (1994). Identification of the gene encoding lipoate-protein ligase A of Escherichia coli: molecular cloning and characterization of the LplA gene and gene product. J. Biol. Chem. 269, 16091-16100.

Perham, R. N. (1991). Domains, motifs, and linkers in 2-oxo acid dehydrogenase multienzyme complexes: a paradigm in the design of a multifunctional enzyme. Biochemistry, 30, 8501-8512.

Perham, R. N. (2000). Swinging arms and swinging domains in multi-functional enzymes: catalytic machines for multistep reactions. Annu. Rev. Biochem. 69, 961-1004.

Quinn, J., Diamond, A. G., Masters, A. K., Brookfield, D. E., Wallis, N. G. \& Yeaman, S. J. (1993). Expression and lipoylation in Escherichia coli of the inner lipoyl domain of the E2 component of the human pyruvate dehydrogenase complex. Biochem. J. 289, 81-85.

Reed, L. J. (1974). Multienzyme complexes. Acc. Chem. Res. 7, 40-46.

Reed, L. J. \& Hackert, M. L. (1990). Structure-function relationships in dihydrolipoamide acyltransferases. J. Biol. Chem. 265, 8971-8974.

Ricaud, P. M., Howard, M. J., Roberts, E. L., Broadhurst, R. W. \& Perham, R. N. (1996). Three-dimensional structure of the lipoyl domain from the dihydrolipoyl succinyltransferase component of the 2-oxoglutarate dehydrogenase multienzyme complex of Escherichia coli. J. Mol. Biol. 264, 179-190.

Wallis, N. G. \& Perham, R. N. (1994). Structural dependence of post-translational modification and reductive acetylation of the lipoyl domain of the pyruvate dehydrogenase multienzyme complex. J. Mol. Biol. 236, 209-216.

Wallis, N. G., Allen, M. D., Broadhurst, R. W., Lessard, I. A. D. \& Perham, R. N. (1996). Recognition of a surface loop of the lipoyl domain underlies substrate channeling in the pyruvate dehydrogenase mutlienzyme complex. J. Mol. Biol. 263, 436-474.

Edited by P. E. Wright 